\title{
On the discrete lot-sizing and scheduling problem with Wagner-Whitin costs
}

Citation for published version (APA):

van Eijl, C. A., \& van Hoesel, C. P. M. (1995). On the discrete lot-sizing and scheduling problem with Wagner-Whitin costs. METEOR, Maastricht University School of Business and Economics. METEOR Research Memorandum No. 018 https://doi.org/10.26481/umamet.1995018

Document status and date:

Published: 01/01/1995

DOI:

10.26481/umamet.1995018

Document Version:

Publisher's PDF, also known as Version of record

\section{Please check the document version of this publication:}

- A submitted manuscript is the version of the article upon submission and before peer-review. There can be important differences between the submitted version and the official published version of record.

People interested in the research are advised to contact the author for the final version of the publication, or visit the DOI to the publisher's website.

- The final author version and the galley proof are versions of the publication after peer review.

- The final published version features the final layout of the paper including the volume, issue and page numbers.

Link to publication

\footnotetext{
General rights rights.

- You may freely distribute the URL identifying the publication in the public portal. please follow below link for the End User Agreement:

www.umlib.nl/taverne-license

Take down policy

If you believe that this document breaches copyright please contact us at:

repository@maastrichtuniversity.nl

providing details and we will investigate your claim.
}

Copyright and moral rights for the publications made accessible in the public portal are retained by the authors and/or other copyright owners and it is a condition of accessing publications that users recognise and abide by the legal requirements associated with these

- Users may download and print one copy of any publication from the public portal for the purpose of private study or research.

- You may not further distribute the material or use it for any profit-making activity or commercial gain

If the publication is distributed under the terms of Article $25 \mathrm{fa}$ of the Dutch Copyright Act, indicated by the "Taverne" license above, 


\title{
On the discrete lot-sizing and scheduling problem with Wagner-Whitin costs
}

\author{
C.A. van Eij $1^{*}$ and C.P.M. van Hoesel ${ }^{\dagger}$
}

\begin{abstract}
We consider the single-item discrete lot-sizing and scheduling problem. We present a partial linear description of the convex hull of feasible solutions that solves this problem in the presence of Wagner-Whitin costs.
\end{abstract}

\section{Introduction}

In recent years a great number of lot-sizing problems have been studied from a polyhedral point of view ( $\mathrm{cf}$. Pochet and Wolsey [5]). Most of the results concern the polyhedral structure of single-item models. Valid inequalities derived for these models have been successfully used in cutting plane algorithms for multi-item problems. Hence, (partial) linear descriptions of the convex hull of feasible solutions of single-item models are a valuable aid in solving lot-sizing problems by methods based on polyhedral combinatorics.

In [4] Pochet and Wolsey study four single-item lot-sizing problems in the presence of Wagner-Whitin costs, i.e., when the unit inventory cost $h_{t}$ and the unit production cost $p_{t}$ satisfy $h_{t}+p_{t} \geq p_{t+1}$ for every period $t$ of the planning interval. For each of these problems, they give a partial linear description of the convex hull of feasible solutions that solves the problem when the costs satisfy the Wagner-Whitin property. These polyhedra involve considerably fewer constraints than in the general cost case.

In this paper we derive a similar result for the single-item discrete lot-sizing and scheduling problem (DLSP). In the following section we formulate the problem and discuss a partial linear description of the convex hull of feasible solutions that solves the problem in the presence of Wagner-Whitin costs. This result is proven in Section 3.

${ }^{*}$ Eindhoven University of Technology, Department of Mathematics and Computing Science, P.O. Box 513, 5600 MB Eindhoven, Netherlands.

${ }^{\dagger}$ University of Limburg, Department of Quantitive Economics, P.O. Box 616, 6200 MD Maastricht, Netherlands. 


\section{The DLSP with Wagner-Whitin costs}

We consider a single-item single-machine production planning problem with a planning horizon of $T$ periods in each of which the production is either zero or at full capacity, say, one unit. This is often a reasonable assumption in short-term production planning, when the time periods are small. The demand in period $t$, denoted by $d_{t}$, is either zero or one, and has to be satisfied in time, i.e., backlogging is not allowed. Furthermore, if production occurs in period $t$, but not in period $t-1$, then a startup has to take place in period $t$, which incurs a cost $f_{t}$. In multi-item problems startup costs also arise when the machine switches from the production of one item to the production of an other item. Apart from startup costs, a production cost $p_{t}$ and a unit inventory cost $h_{t}$ are given for each period $t$. Now DLSP is the problem of determining a production schedule that satisfies the above restrictions at minimum costs.

The problem can be mathematically formulated using two types of binary variables: $x_{t}$, which indicates whether production occurs in period $t$ or not, and $y_{t}$, which equals one if a startup occurs in period $t$ and zero otherwise. For notational convenience we write $x_{t_{1}, t_{2}}$ instead of $\sum_{t=t_{1}}^{t_{2}} x_{t}, d_{t_{1}, t_{2}}$ instead of $\sum_{t=t_{1}}^{t_{2}} d_{t}$, etc. Furthermore, we denote by $s_{i}$ the $i$ th demand period in $\{1, \ldots, T\}$, thus, $d_{s_{i}}=1$ and $d_{1, s_{i}}=i$. Now DLSP is modelled as follows:

$$
\begin{array}{lll}
\text { (DLSP }) & \min \sum_{t=1}^{T}\left(f_{t} y_{t}+c_{t} x_{t}\right) & \\
\text { s.t. } & x_{1, t} \geq d_{1, t} & (1 \leq t<T) \\
& x_{1, T}=d_{1, T} & \\
& y_{t} \leq x_{t} \leq x_{t-1}+y_{t} & \left(1 \leq t \leq T, x_{0}=0\right) \\
& x_{t}, y_{t} \in\{0,1\} & (1 \leq t \leq T)
\end{array}
$$

In (1) we have $c_{t}=p_{t}+h_{t, T}$. The latter term is obtained by expressing the inventory costs as $\sum_{t=1}^{T} h_{t} \cdot\left(x_{1, t}-d_{1, t}\right)=\sum_{t=1}^{T} h_{t, T} x_{t}$ minus a constant, which is omitted from the objective function. Inequalities (2) yield that the total production up to period $t$ equals at least the total demand up to this period. Furthermore, overproduction is prohibited by (3). Constraints (4) force that a startup takes place in period $t$ if production occurs in this period but not in the preceding one. Moreover, these constraints prevent that a startup occurs in a period without production.

Although the single-item DLSP is polynomially solvable, the convex hull of the set of feasible solutions of (2)-(5) is not known explicitly. Van Hoesel [2] discusses several classes of facetdefining inequalities. Magnanti and Vachani [3] and Sastry [6] derive inequalities for a slightly more general problem in which also setup costs are involved. 
The following inequalities are adapted from the interval left supermodular inequalities derived by Constantino ([1], Section 2.2) for the capacitated lot-sizing problem with startup costs. This problem is a generalization of DLSP in which the production in period $t$ can attain any value between zero and the available capacity in this period.

Lemma 1 Let $t \in\{1, \ldots, T\}$ and $j \in\left\{0, \ldots, d_{t+1, T}\right\}$. Then all feasible solutions of DLSP satisfy

$$
x_{1, t}+\sum_{i=1}^{j}\left(x_{t+i}+y_{t+i+1, s_{d_{1, t}+i}}\right) \geq d_{1, t}+j .
$$

Note that there exist only $O\left(T d_{1, T}\right)$ constraints of this form. Before proving the validity of $(6)$, let us briefly explain the idea behind these inequalities. Observe that $x_{t+i}+y_{t+i+1, s_{d_{1, t}+i}}$ is nonnegative and integral for any feasible solution $(y, x)$ of DLSP. Moreover, $x_{t+i}+y_{t+i+1, s_{d_{1, t}+i}}=0$ if and only if no production occurs in the interval $\left\{t+i, \ldots, s_{d_{1, t}+i}\right\}$. In this case the stock at the end of period $t$ must be at least $d_{t+1, s_{d_{1, t}+i}}-i+1=1$. Rewrite (6) as $x_{1, t}-d_{1, t} \geq \sum_{i=1}^{j}\left(1-x_{t+i}-y_{t+i+1, s_{d_{1, t}+i}}\right)$ and observe that the new lefthand side denotes the stock at the end of period $t$. Now one immediately sees that this constraint forces the stock at the end of period $t$ to be at least one if no production occurs in the interval $\left\{t+i, \ldots, s_{d_{1, t}+i}\right\}$.

Proof of Lemma 1. First, note that inequalities (2) are a special case of (6) (take $j=0$ ). Hence, for every $t$ and $j=0,(6)$ is valid. Suppose that (6) is not valid for some $t$ and $j>0$, where $j$ is minimal. Let $(y, x)$ be a solution of DLSP violating this constraint. Since the constraint obtained by replacing $j$ by $j-1$ is valid, we have

$$
\begin{aligned}
d_{1, t}+j & >x_{1, t}+\sum_{i=1}^{j}\left(x_{t+i}+y_{t+i+1, s_{d_{1, t}+i}}\right) \\
& =x_{1, t}+\sum_{i=1}^{j-1}\left(x_{t+i}+y_{t+i+1, s_{d_{1, t}+i}}\right)+x_{t+j}+y_{t+j+1, s_{d_{1, t}+j}} \\
& \geq d_{1, t}+j-1+x_{t+j}+y_{t+j+1, s_{d_{1, t}+j}} .
\end{aligned}
$$

Thus, since $(y, x)$ is integral, $x_{t+j}+y_{t+j+1, s_{d_{1, t}+j}}=0$, which implies that there is no production in $\left\{t+j, \ldots, s_{d_{1, t}+j}\right\}$. Therefore, to satisfy the production requirements (2) we must have $x_{1, t+j-1} \geq d_{1, t}+j$, which contradicts the fact that $(y, x)$ violates $(6)$ for the given choices of $t$ and $j$.

Recall that the costs are said to satisfy the Wagner-Whitin property if $h_{t}+p_{t} \geq p_{t+1}$, or, equivalently, $c_{t} \geq c_{t+1}$ for all $t$. With Wagner-Whitin costs there always exists an optimal solution satisfying the zero-inventory property, i.e., when a new production batch is started in period $t$, the stock at the end of period $t-1$ is zero.

Denote by RDLSP the LP-relaxation of DLSP where inequalities (6) replace (2). We claim that the following holds: 
Theorem 2 For cost functions that satisfy the Wagner-Whitin property, the objective value of RDLSP equals the objective value of DLSP.

When $c_{t}$ strictly decreases in $t$, an even stronger result can be proven, namely, that RDLSP solves DLSP.

Theorem 3 If (1) satisfies $c_{t}>c_{t+1}$ for every period t, then any optimal solution of $R D L S P$ is a convex combination of feasible solutions of DLSP, i.e., the set of optimal solutions of RDLSP has integral extreme points.

The proof of the above theorems is postponed until the following section.

Our purpose is to develop a branch-and-cut algorithm for solving multi-item problems. Due to the above result, the $O\left(T d_{1, T}\right)$ constraints of type $(6)$ are expected to yield strong cutting planes. Furthermore, we will study the effectiveness of these inequalities for problems with more complicating features such as startup times.

\section{Proof of the theorems}

The major part of this section deals with the proof of Theorem 3. Therefore, assume that $c_{t}>c_{t+1}$ for every $t$. The proof uses a partitioning of a solution $(y, x)$ of RDLSP into a set of batches $\mathcal{B}$, where a batch $B=\left\{p^{B}, \ldots, q^{B}\right\}$ is identified with the partial solution $\left(y^{B}, x^{B}\right)$ defined by

$$
\begin{aligned}
& y^{B}=\left(\begin{array}{lllllll}
0 & \ldots & 1 & 0 & \ldots & 0 & 0
\end{array}\right) \\
& x^{B}=\frac{(0 \ldots 011 \ldots 10 \ldots 0)}{1 \quad p^{B} \quad q^{B} \quad T}
\end{aligned}
$$

Furthermore, a value $b^{B}, 0<b^{B} \leq 1$, is attached to every batch $B$ such that $(y, x)=$ $\sum_{B \in \mathcal{B}} b^{B}\left(y^{B}, x^{B}\right)$. We say that $\mathcal{B}$ satisfies the partitioning condition if

$$
\forall i \in\left\{1, \ldots, d_{1, T}\right\} \sum_{B \in \mathcal{B}: s_{i} \in I^{B}} b^{B}=1,
$$

where $I^{B}$ consists of the first $|B|$ demand periods in $\left\{p^{B}, \ldots, T\right\}$.

The proof of Theorem 3 consists of the following two steps. First, we prove that the partitioning condition is a sufficient condition for $(y, x)$ to be a convex combination of solutions of DLSP (Lemma 4). Second, we present a greedy algorithm that partitions any optimal solution $\left(y^{*}, x^{*}\right)$ of RDLSP into a set of batches $\mathcal{B}$ with values $b^{B}, B \in \mathcal{B}$, such that $\left(y^{*}, x^{*}\right)=$ $\sum_{B \in \mathcal{B}} b^{B}\left(y^{B}, x^{B}\right)$ and the partitioning condition is satisfied. Combining these results yields that all extreme points of the set of optimal solutions of RDLSP are integral.

Lemma 4 Given a set of batches $\mathcal{B}$ with values $b^{B}, 0<b^{B} \leq 1, B \in \mathcal{B}$, such that (7) is satisfied. Then $(y, x):=\sum_{B \in \mathcal{B}} b^{B}\left(y^{B}, x^{B}\right)$ is a convex combination of solutions of DLSP. 
Proof. The lemma is proved by induction on the number of batch-pairs $(B, D)$ in $\mathcal{B}$ with intersecting demand sets $I^{B}$ and $I^{D}$, i.e., $I^{B} \cap I^{D} \neq \emptyset$.

If no two batches have intersecting demand set, then each batch $B$ in $\mathcal{B}$ has value $b^{B}=1$ by (7), and the lemma follows immediately.

If there are batches with intersecting demand set, then, by ( 7$)$, there is a batch $B$ with value $b^{B}<1$. We construct a subset $\mathcal{D}$ of $\mathcal{B}$ such that the demand sets of the batches in $\mathcal{D}$ form a partition of the demand periods. First, we take a batch the demand set of which contains the first demand period $s_{1}$. Suppose that we have found a set of batches $\mathcal{D}$ such that the corresponding demand sets form a partition of the first $j<d_{1, T}$ demand periods. Then there exists a batch $D \in \mathcal{B} \backslash \mathcal{D}$ such that the demand set $I^{D}$ contains $s_{j+1}$ but not $s_{j}$. This follows from

$$
\sum_{B \in \mathcal{B} \backslash \mathcal{D}: s_{j+1} \in I^{B}} b^{B}=\sum_{B \in \mathcal{B}: s_{j+1} \in I^{B}} b^{B}=1=\sum_{B \in \mathcal{B}: s_{j} \in I^{B}} b^{B}>\sum_{B \in \mathcal{B} \backslash \mathcal{D}: s_{j} \in I^{B}} b^{B} .
$$

The demand set of $D$ is $\left\{s_{j+1}, \ldots, s_{j^{\prime}}\right\}$ for some $j^{\prime} \in\left\{j+1, \ldots, d_{1, T}\right\}$. Addition of $D$ to $\mathcal{D}$ gives a partition of the demand periods $\left\{s_{1}, \ldots, s_{j^{\prime}}\right\}$. We proceed in this way until $\mathcal{D}$ is a partition of $\left\{s_{1}, \ldots, s_{d_{1, T}}\right\}$. By construction, the integral vector $\left(y^{\prime}, x^{\prime}\right):=\sum_{B \in \mathcal{D}}\left(y^{B}, x^{B}\right)$ is a feasible solution of DLSP.

Set $\bar{b}=\min \left\{b^{B} \mid B \in \mathcal{D}\right\}$ and define $\overline{\mathcal{B}}=\mathcal{B} \backslash\left\{B \in \mathcal{D} \mid b^{B}=\bar{b}\right\}$. Note that $\bar{b}<1$. Furthermore, set $\bar{b}^{B}=\left(b^{B}-\bar{b}\right) /(1-\bar{b})$ for $B \in \overline{\mathcal{B}} \cap \mathcal{D}$ and $\bar{b}^{B}=b^{B} /(1-\bar{b})$ for $B \in \overline{\mathcal{B}} \backslash \mathcal{D}$. Let $i \in\left\{1, \ldots, d_{1, T}\right\}$. Since there is exactly one batch $B \in \mathcal{D}$ such that $s_{i} \in I^{B}$, we have

$$
\sum_{B \in \overline{\mathcal{B}}: s_{i} \in I^{B}} \bar{b}^{B}=\sum_{B \in \mathcal{D}: s_{i} \in I^{B}} \frac{b^{B}-\bar{b}}{1-\bar{b}}+\sum_{B \in \mathcal{B} \backslash \mathcal{D}: s_{i} \in I^{B}} \frac{b^{B}}{1-\bar{b}}=\frac{\sum_{B \in \mathcal{B}: s_{i} \in I^{B}} b^{B}-\bar{b}}{1-\bar{b}}=1 .
$$

Hence, $\overline{\mathcal{B}}$ satisfies the partitioning condition. Since $|\overline{\mathcal{B}}|<|\mathcal{B}|$, the number of pairwise intersecting demand sets in $\overline{\mathcal{B}}$ is less than the number of pairwise intersecting demand sets in $\mathcal{B}$. Now the induction hypothesis yields that $\left(y^{\prime \prime}, x^{\prime \prime}\right):=\sum_{B \in \overline{\mathcal{B}}} \bar{b}^{B}\left(y^{B}, x^{B}\right)$ is a convex combination of integral solutions. Thus, so is $(y, x)=\bar{b}\left(y^{\prime}, x^{\prime}\right)+(1-\bar{b})\left(y^{\prime \prime}, x^{\prime \prime}\right)$.

From the above lemma it follows that, in order to prove Theorem 3, it suffices to show that any optimal solution $\left(y^{*}, x^{*}\right)$ of RDLSP can be partitioned into a set of batches $\mathcal{B}$ with values $b^{B}, B \in \mathcal{B}$, such that $\left(y^{*}, x^{*}\right)=\sum_{B \in \mathcal{B}} b^{B}\left(y^{B}, x^{B}\right)$ and the partitioning condition is satisfied. In the sequel, let $\left(y^{*}, x^{*}\right)$ denote an optimal solution of RDLSP. We claim that the following algorithm provides a set of batches $\mathcal{B}$ with the desired properties. 


\section{begin CONSTRUCT_BATCHES}

for $t=1$ to $T$ do begin $\bar{x}_{t}:=x_{t}^{*} ; \bar{y}_{t}:=y_{t}^{*} ; \bar{d}_{t}:=d_{t}$ end;

$\left\{\bar{x}_{t}\right.$ is called the residual production, etc. $\}$

$\mathcal{D}:=\emptyset$;

while $\bar{x}_{1, T}>0$ do

begin

$q^{D}:=$ last period with positive residual production;

$p^{D}:=$ last period in $\left\{1, \ldots, q^{D}\right\}$ with positive residual startup;

$D:=\left\{p^{D}, \ldots, q^{D}\right\}$;

$J^{D}:=$ set of demand periods with positive residual demand in $\left\{p^{D}, \ldots, T\right\}$;

$b^{D}:=\min \left\{\bar{y}_{p^{D}}, \min _{t \in D} \bar{x}_{t}, \min _{t \in J^{D}} \bar{d}_{t}\right\}$;

$\bar{y}_{p^{D}}:=\bar{y}_{p^{D}}-b^{D}$;

for $t \in D$ do $\bar{x}_{t}:=\bar{x}_{t}-b^{D}$;

for $t \in J^{D}$ do $\bar{d}_{t}:=\bar{d}_{t}-b^{D}$;

$\mathcal{D}:=\mathcal{D} \cup\{D\}$

end;

end.

Observe that $\bar{x}_{t}, \bar{y}_{t}$, and $\bar{d}_{t}$ are non-increasing and nonnegative during the execution of the algorithm. Moreover, the residual demands $\bar{d}_{s_{i}}$ are non-increasing in $i$. It is also easily seen that $\bar{x}_{t} \leq \bar{y}_{t}+\bar{x}_{t-1}$ and $\bar{y}_{t} \leq \bar{x}_{t}$ hold for all $t$. Therefore, $\bar{x}_{q^{D}}=\min _{t \in D} \bar{x}_{t}$, and if $J^{D} \neq \emptyset$, then $\bar{d}_{\bar{s}}=\min _{t \in J^{D}} \bar{d}_{t}$, where $\bar{s}$ denotes the last period with positive residual demand. We will prove that during the execution of the algorithm the following invariant holds:

( $\left.\mathrm{I}_{1}\right) \forall_{t \in\{1, \ldots, T\}} x_{t}^{*}=\bar{x}_{t}+\sum_{B \in \mathcal{D}: t \in B} b^{B}$

(I $\left.\mathrm{I}_{2}\right) \forall_{t \in\{1, \ldots, T\}} y_{t}^{*}=\bar{y}_{t}+\sum_{B \in \mathcal{D}: t=p^{B}} b^{B}$

(I $\mathrm{I}_{3} \forall_{i \in\left\{1, \ldots, d_{1, T}\right\}} 1=\bar{d}_{s_{i}}+\sum_{B \in \mathcal{D}: s_{i} \in J^{B}} b^{B}$

(I $\mathrm{I}_{4} \forall_{B \in \mathcal{D}} \quad\left|J^{B}\right|=|B|$

(I $\forall_{5} \forall_{t \in\{1, \ldots, T-1\}} \bar{x}_{1, t} \geq \bar{d}_{1, t}$ and $\bar{x}_{1, T}=\bar{d}_{1, T}$.

Note that for $t<p^{B}$, the residual values are equal to the original values, i.e., $\bar{x}_{t}=x_{t}^{*}, \bar{y}_{t}=y_{t}^{*}$, and $\bar{d}_{t}=d_{t}$.

Suppose that $\left(\mathrm{I}_{1}\right)-\left(\mathrm{I}_{5}\right)$ hold during the execution of the algorithm. At termination of the algorithm we have $\bar{x}_{t}=\bar{y}_{t}=0$ and, by $\left(\mathrm{I}_{5}\right), \bar{d}_{t}=0$ for all $t$. Hence, by $\left(\mathrm{I}_{1}\right)$ and $\left(\mathrm{I}_{2}\right)$, the set of batches $\mathcal{D}$ provided by ConstruCT_BATChes satisfies $\left(y^{*}, x^{*}\right)=\sum_{B \in \mathcal{D}} b^{B}\left(y^{B}, x^{B}\right)$. Moreover, from $\left(\mathrm{I}_{4}\right)$ it follows that $J^{B}, B \in \mathcal{B}$, can be identified with $I^{B}$, i.e., the set of 
the first $|B|$ demand periods in $\left\{p^{B}, \ldots, T\right\}$. Together with $\left(\mathrm{I}_{3}\right)$, this implies that the set $\mathcal{D}$ satisfies the partitioning condition. Now Lemma 4 yields that $\left(y^{*}, x^{*}\right)$ is a convex combination of feasible solutions of DLSP. Thus, the validity of the invariant during the execution of the algorithm implies the validity of Theorem 3 .

The invariant is easily checked to hold initially. We will prove that if the invariant holds at the beginning of an iteration, then it also holds at the end of that iteration. In the sequel the current iteration is the one for which validity of the invariant is proven. We denote the batch defined in the current iteration by $D$. The set of batches that are constructed in previous iterations is denoted by $\mathcal{D}$. Now $\left(\mathrm{I}_{1}\right)-\left(\mathrm{I}_{3}\right)$ are easily checked to hold at the end of the current iteration, and $\left(\mathrm{I}_{5}\right)$ follows from $\left(\mathrm{I}_{4}\right)$. The latter holds at the end of the current iteration if $\left|J^{D}\right|=|D|$. Hence, we are left with the proof of $\left|J^{D}\right|=|D|$.

Proof of $\left|J^{D}\right|=|D|$.

We first show that $\left|J^{D}\right|>|D|$ implies that $\left(y^{*}, x^{*}\right)$ is not optimal. Next, we show that if $\left|J^{D}\right|<|D|$, then $\left(y^{*}, x^{*}\right)$ violates a constraint of type $(6)$. Both results contradict the assumption that $\left(y^{*}, x^{*}\right)$ is an optimal solution of RDLSP, which leads to the conclusion that $\left|J^{D}\right|=|D|$.

Part 1: $\left|J^{D}\right| \leq|D|$.

Assume that $\left|J^{D}\right|>|D|$. We claim that in this case we can move an amount $\epsilon>0$ from the production in period $q^{D}$ to period $q^{D}+1$ while maintaining feasibility. Since $c_{q^{D}}>c_{q^{D}+1}$, this yields a cheaper solution than $\left(y^{*}, x^{*}\right)$, which contradicts the optimality of $\left(y^{*}, x^{*}\right)$. In order to prove our claim, it suffices to show that the following constraints have positive slack, i.e., they are not satisfied at equality:

(i) $x_{q^{D}}^{*} \geq 0$

(ii) $x_{q^{D}+1}^{*} \leq 1$

(iii) $x_{q^{D}+1}^{*} \leq y_{q^{D}+1}^{*}+x_{q^{D}}^{*}$

(iv) $\forall_{t, j: t+j=q^{D}} x_{1, t}^{*}+\sum_{i=1}^{j}\left(x_{t+i}^{*}+y_{t+i+1, s_{d_{1, t}+i}}^{*}\right) \geq d_{1, t}+j$.

By definition of $q^{D}$, we have $x_{q^{D}}^{*} \geq \bar{x}_{q^{D}}>0$. For the proof of $x_{q^{D}+1}^{*}<1$, we use the following important observation: if period $s$ has positive residual demand in the current iteration, then $s \in J^{B}$ for every batch $B \in \mathcal{D}$ with $p^{B} \leq s$. Now let $s^{\prime}$ be the first demand period after $q^{D}$. Then $\bar{d}_{s^{\prime}}>0$, since $\left|J^{D}\right|>|D|$. Hence, if $B \in \mathcal{D}$ satisfies $q^{D}+1 \in B$, then $s^{\prime} \in J^{B}$. Together with $\bar{x}_{q^{D}+1}=0$, this yields

$$
x_{q^{D}+1}^{*}=x_{q^{D}+1}^{*}-\bar{x}_{q^{D}+1} \stackrel{\left(\mathrm{I}_{1}\right)}{=} \sum_{B: q^{D}+1 \in B} b^{B} \leq \sum_{B: s^{\prime} \in J^{D}} b^{B}=1-\bar{d}_{s^{\prime}}<1 .
$$


In order to show that (iii) is not satisfied at equality, notice that whenever $\bar{x}_{q^{D}+1}$ decreases in an iteration, one of the variables $\bar{x}_{q^{D}}$ or $\bar{y}_{q^{D}+1}$ decreases by the same amount. At the beginning of the current iteration, strict inequality holds since $0=\bar{x}_{q^{D}+1}<\bar{x}_{q^{D}}$.

Finally, consider a constraint (6) such that $t+j=q^{D}$. Now

$$
\begin{aligned}
& x_{1, t}^{*}+\sum_{i=1}^{j}\left(x_{t+i}^{*}+y_{t+i+1, s_{d_{1, t}+i}^{*}}^{*}\right) \\
& \stackrel{\left(\mathrm{I}_{1}\right),\left(\mathrm{I}_{2}\right)}{=} \quad x_{1, t}^{*}+\sum_{i=1}^{j}\left(\bar{x}_{t+i}+\bar{y}_{t+i+1, s_{d_{1, t}+i}}\right)+\sum_{i=1}^{j} \sum_{B \in \mathcal{D}: q^{B} \geq t+i, p^{B} \leq s_{d_{1, t}+i}} b^{B} \\
& \geq \quad x_{1, t}^{*}+\bar{x}_{t+1, t+j}+\bar{y}_{t+2, p^{D}}+\sum_{i=1}^{j} \sum_{B \in \mathcal{D}: q^{B} \geq t+i, p^{B} \leq s_{d_{1, t}+i}} b^{B} \\
& \quad{ }^{(*)} \quad x_{1, t}^{*}+\bar{x}_{t+1, t+j}+\bar{y}_{t+2, p^{D}}+\sum_{i=1}^{j} \sum_{B \in \mathcal{D}: s_{d_{1, t}+i \in J^{B}}} b^{B} \\
& \stackrel{\left(\mathrm{I}_{3}\right)}{=} \quad x_{1, t}^{*}+\bar{x}_{t+1, q^{D}}+\bar{y}_{t+2, p^{D}}+\sum_{i=1}^{j}\left(1-\bar{d}_{s_{d_{1, t}+i}}\right),
\end{aligned}
$$

where $(*)$ holds because $s_{d_{1, t}+i} \in J^{B}$ implies $p^{B} \leq s_{d_{1, t}+i}$, and $t+i \leq t+j=q^{D} \leq q^{B}$. In order to show that strict inequality holds for the constraint under consideration, we distinguish two cases. First, suppose that $p^{D}>t+1$. Then, since $\bar{y}_{p D}>0$ and $\bar{x}_{1, q^{D}}=\bar{x}_{1, T}$, we have

$$
\begin{aligned}
& x_{1, t}^{*}+\bar{x}_{t+1, q^{D}}+\bar{y}_{t+2, p^{D}}+\sum_{i=1}^{j}\left(1-\bar{d}_{s_{d_{1, t}+i}}\right) \\
& \quad>\bar{x}_{1, T}+\sum_{i=1}^{j}\left(1-\bar{d}_{s_{d_{1, t}+i}}\right) \stackrel{\left(\mathrm{I}_{5}\right)}{\geq} \bar{d}_{1, s_{d_{1, t}+j}}+\sum_{i=1}^{j}\left(1-\bar{d}_{s_{d_{1, t}+i}}\right)=\bar{d}_{1, t}+j=d_{1, t}+j .
\end{aligned}
$$

If $p^{D} \leq t+1$, then the last period with positive residual demand $\bar{s}>s_{d_{1, t}+j}$, hence, ( $\left.\mathrm{I}_{5}\right)$ yields that $\bar{x}_{1, q^{D}}=\bar{x}_{1, T}=\bar{d}_{1, \bar{s}}>\bar{d}_{1, s_{d_{1, t}+j}}$. We have

$$
\begin{aligned}
& x_{1, t}^{*}+\bar{x}_{t+1, q^{D}}+\bar{y}_{t+2, p^{D}}+\sum_{i=1}^{j}\left(1-\bar{d}_{s_{d_{1, t}+i}}\right) \stackrel{(*)}{\geq} \bar{x}_{1, q^{D}}+\sum_{i=d_{1, p^{D}-1}+1}^{d_{1, t}+j}\left(1-\bar{d}_{s_{i}}\right) \\
& >\bar{d}_{1, s_{d_{1, t}+j}}+\sum_{i=d_{1, p^{D}-1}+1}^{d_{1, t}+j}\left(1-\bar{d}_{s_{i}}\right)=d_{1, p^{D}-1}+d_{1, t}+j-d_{1, p^{D}-1}=d_{1, t}+j,
\end{aligned}
$$

where $(*)$ follows from the validity of $\left(\mathrm{I}_{1}\right)$ for every period in $\left\{p^{D}, \ldots, t\right\}$, the validity of $\left(\mathrm{I}_{3}\right)$ for $i \in\left\{d_{1, p^{D}-1}+1, \ldots, d_{1, t}\right\}$, and the trivial observations that for any $B \in \mathcal{D}$ we have $q^{B} \geq q^{D}>t$ and the number of periods with positive residual demand in $\left\{p^{B}, \ldots, t\right\}$ is at most $t-p^{B}+1$.

We conclude that none of the constraints (i)-(iv) is satisfied at equality, which establishes the validity of $\left|J^{D}\right| \leq|D|$. 
Part 2: $\left|J^{D}\right| \geq|D|$.

Suppose that $\left|J^{D}\right|<|D|$. We claim that in this case the constraint of type (6) with $t=p^{D}-1$ and $j=\left|J^{D}\right|$ is violated by $\left(y^{*}, x^{*}\right)$. Note that this is trivial when $\left|J^{D}\right|=0$, so we will assume that $\left|J^{D}\right|>0$. In the proof we use the following observation:

$$
\forall_{t \in\left\{p^{D}+1, \ldots, T\right\}} \quad \bar{y}_{t}=0 .
$$

Note that for $t \in\left\{p^{D}+1, \ldots, q^{D}\right\}$ this is by choice of $p^{D}$. For $t \in\left\{q^{D}+1, \ldots, T\right\}$ this follows from $\bar{y}_{t} \leq \bar{x}_{t}$.

Also note that $s_{d_{1, p^{D}-1}+\left|J^{D}\right|}$ the last period with positive residual demand, hence, the righthand side of the constraint under consideration equals $d_{1, t}+\left|J^{D}\right|=d_{1, \bar{s}}$. We have

$$
\begin{aligned}
& x_{1, p^{D}-1}^{*}+\sum_{i=1}^{\left|J^{D}\right|}\left(x_{p^{D}-1+i}^{*}+y_{p^{D}-1+i+1, s_{d_{1, p^{D}-1}+i}^{*}}^{*}\right) \\
& \stackrel{\left(\mathrm{I}_{1}\right),\left(\mathrm{I}_{2}\right)}{=} \bar{x}_{1, p^{D}-1+\left|J^{D}\right|}+\sum_{i=1}^{\left|J^{D}\right|} \bar{y}_{p^{D}-1+i+1, s_{d_{1, p^{D}-1}+i}}+\sum_{i=1}^{\left|J^{D}\right|} \sum_{B \in \mathcal{D}: q^{B} \geq p^{D}-1+i, p^{B} \leq s_{d_{1, p^{D}-1}+i}} b^{B} \\
& \stackrel{(8)}{=} \quad \bar{x}_{1, p^{D}-1+\left|J^{D}\right|}+\sum_{i=1}^{\left|J^{D}\right|} \sum_{B \in \mathcal{D}: q^{B} \geq p^{D}} b_{-1+i, p^{B} \leq s_{d_{1, p^{D}-1}}+i} b^{B} \\
& \stackrel{(*)}{\leq} \quad \bar{x}_{1, p^{D}-1+\left|J^{D}\right|}+\sum_{i=1}^{\left|J^{D}\right|} \sum_{B \in \mathcal{D}: s_{d_{1, p^{D}}-1}+i \in J^{B}} b^{B} \\
& \stackrel{\left(\mathrm{I}_{3}\right)}{=} \quad \bar{x}_{1, p^{D}-1+\left|J^{D}\right|}+\sum_{i=1}^{\left|J^{D}\right|}\left(1-\bar{d}_{s_{d_{1, p^{D}-1}}+i}\right) \stackrel{(\dagger)}{<} d_{1, \bar{s}} \text {. }
\end{aligned}
$$

Note that in the current iteration all demand periods in $\left\{p^{D}, \ldots, \bar{s}\right\}$ have positive residual demand. Thus, for each $B \in \mathcal{B}$ with $p^{B} \leq s_{d_{1, p}{ }_{-1}+i}, i \leq\left|J^{D}\right|$, we have $s_{d_{1, p^{D}-1}+i} \in J^{B}$. This shows the validity of $(*)$. Moreover, the assumption that $\left|J^{D}\right|<|D|$ yields that $p^{D}-1+\left|J^{D}\right|<q^{D}$, hence, by definition of $q^{D}$ and $\left(\mathrm{I}_{5}\right)$, we have $\bar{x}_{1, p^{D}-1+\left|J^{D}\right|}<\bar{x}_{1, q^{D}}=\bar{d}_{1, \bar{s}}$. From this the validity of $(\dagger)$ immediately follows.

This concludes the proof of $\left|J^{D}\right|=|D|$ and, hence, the proof of Theorem 3 .

As a corollary we can prove Theorem 2 as follows. For arbitrary $\epsilon>0$ the cost function $c_{t}^{\prime}:=c_{t}+(T-t) \epsilon$ satisfies the requirements of Theorem 3 . Therefore, for every $\epsilon>0$ there exists an optimal solution of RDLSP that is an integral extreme point. Since the objective function is continuous in $\epsilon$, there must be an integer optimal solution of RDLSP for $\epsilon=0$. However, we do not necessarily find that for $\epsilon=0$ all extreme points of the set of optimal solutions of RDLSP are integral. 


\section{References}

[1] M.F. Constantino, A polyhedral approach to production planning models: start-up costs and times, upper and lower bounds on production, Ph.D. Thesis, Faculté des Sciences, Université Catholique de Louvain, Louvain-la-Neuve, 1995.

[2] S. van Hoesel, Models and algorithms for single-item lot sizing problems, Ph.D. Thesis, Econometrisch Instituut, Erasmus Universiteit, Rotterdam, 1991.

[3] T.L. Magnanti, R. Vachani, A strong cutting plane algorithm for production scheduling with changeover costs, Operations Research 38 (1990), 456-473.

[4] Y. Pochet, L.A. Wolsey, Polyhedra for lot-sizing with Wagner-Whitin costs, Math. Programming 67 (1994), 297-323.

[5] Y. Pochet, L.A. Wolsey, Algorithms and reformulations for lot sizing problems, in: W. Cook, L. Lovász, P.D. Seymour (eds.), Combinatorial optimization, DIMACS series in discrete mathematics and theoretical computer science 20, 1995, 245-293

[6] T. Sastry, Polyhedral structure of the product cycling problem with changeover costs, Ph.D. Thesis, Sloan School of Management, MIT, Cambridge, USA, 1990. 